\title{
MUERTES EVITABLES ASOCIADAS A LA IMPLEMENTACIÓN DE INFRAESTRUCTURA VERDE URBANA EN QUITO
}

\section{AVOIDABLE DEATHS ASSOCIATED WITH THE IMPLEMENTATION OF URBAN GREEN INFRASTRUCTURE IN QUITO}

\section{Sebastián Rodríguez¹ , Alexandra Mena ${ }^{1}$ Daniel Garzón², Francisco Ramírez ${ }^{3}$ \& David Chuquer-Solá ${ }^{*}$}

Recibido: 19 de junio 2020 / Aceptado: 20 de noviembre 2020 DOI: $10.26807 /$ ia.v9i1.173

Palabras claves: Calidad del aire, Evaluación evaluación de impactos en salud, Infraestructura Verde Urbanainfraestructura verde urbana, Metodología AP-HRA, Muertes Evitables.

Keywords: Air quality, AP-HRA methodology, Avoidable deaths, Health risk assessment, Urban green infrastructure.

\footnotetext{
1 Pontificia Universidad Católica del Ecuador, Facultad de Ciencias Humanas, Quito-Ecuador. (mrodriguez907@puce.edu.ec ; amena001@puce.edu.ec )

2 Universidad San Francisco de Quito, Escuela de Medicina, Ecuador, Quito (dgarzonc@usfq.edu.ec ). 3 Pontificia Universidad Católica del Ecuador, Facultad de Arquitectura, Diseño y Artes, Quito-Ecuador. (rframirez@puce.edu.ec).

4 Pontificia Universidad Católica del Ecuador, Facultad de Ciencias Exactas y Naturales, Quito-Ecuador. ("correspondencia: dchuquer295@puce.edu.ec ).
} 


\section{RESUMEN}

En las últimas décadas, el desarrollo económico de Quito ha venido acompañado con un deterioro en su calidad del aire, principalmente por el aumento progresivo de su flota vehicular. Ante ello, la autoridad ambiental de la zona, registra concentraciones que superan las normas nacionales e internacionales de calidad del aire en los parámetros de $\mathrm{PM}_{10}, \mathrm{PM}_{2,5}$ y $\mathrm{O}_{3}$. Ante este escenario, la implementación de infraestructura verde urbana (IVU) emerge como una alternativa para el mejoramiento de la calidad del aire en microambientes, sin embargo sus costos limitan su aplicación. El trabajo presentado, estima, mediante la metodología AP-HRA, los efectos económicos y en salud pública que se obtendría producto de una implementación agresiva de IVU en ocho parroquias de Quito. Los resultados generados son alentadores dado que una reducción del 30 \% en $\mathrm{PM}_{10}$ y $\mathrm{O}_{3}$ y 1 \% en $\mathrm{PM}_{2,5}$, podría evitar 190 muertes anualmente en la zona de estudio, con un beneficio económico para la sociedad de 139,7 millones de USD. Las estimaciones presentadas, pueden servir de herramienta para los tomadores de decisión con el fin de instrumentar políticas públicas que promuevan la implementación de IVU en espacios públicos y privados.

\section{ABSTRACT}

In recent decades, the economic development of Quito has been accompanied by deterioration in its air quality, mainly due to the progressive increase in its vehicle fleet. Given this, the environmental authority of the area registers concentrations that exceed national and international air quality standards in the parameters of $\mathrm{PM}_{10}, \mathrm{PM}_{2.5}$, and $\mathrm{O}_{3}$. With this scenario, the implementation of urban green infrastructure ( $U \mathrm{Gl})$ emerges as an alternative for improving air quality in microenvironments; however, its costs limit its application. The work presented estimates, through the AP-HRA methodology, the economic and public health effects that would be obtained as a result of the aggressive implementation of UGI in eight parishes in Quito. Results generated are encouraging given that a $30 \%$ reduction in $\mathrm{PM}_{10}$ and $\mathrm{O}_{3}$ and $1 \%$ in $\mathrm{PM}_{2.5}$, could prevent 
190 deaths annually in the studied area, with an economic benefit for the society of USD 139.7 million. The estimates presented can serve as a tool for decision-makers to implement public policies that promote the implementation of UGI in public and private spaces.

\section{INTRODUCCIÓN}

Durante el 2012, en el Ecuador se estimó que existieron once muertes por cada cien mil habitantes atribuibles directamente a la mala calidad del aire (WHO, 2016a). Esto evidencia un problema de salud pública digno de atención.

La incidencia de los contaminantes y su asociación con la mortalidad ha sido estudiada por diversos autores (Hoek et al., 2013; Künzli et al., 2000; Turner et al., 2016; Woodruff et al., 2008). Producto de ello se han obtenido las Funciones Exposición Respuesta (FER) y Riesgos Relativos (RR) correspondientes a varios contaminantes (Echániz Pellicer et al., 2011). El riesgo de que la contaminación atmosférica afecte la salud puede aumentar en determinadas poblaciones vulnerables debido a inequidades socioambientales. De esta manera, la susceptibilidad a impactos negativos a la salud también depende de la capacidad de resistencia de la población, determinado por conductas individuales o colectivas de prevención y precaución (Jiménez et al., 2015). En el Ecuador, de acuerdo con datos del 2019, las enfermedades isquémicas del corazón constituyen la principal causa de muerte con 12,0 $\%$ del total $(n=4852)$ y $11,3 \%(n=$ 3722) para hombres y mujeres respectivamente. De la misma manera las enfermedades infecciosas del aparato respiratorio corresponden a la quinta causa de muerte más frecuente en hombres y mujeres con 6,2 $\%(n=2050)$ y $5,0 \%(n=2046)$ respectivamente. Finalmente, la octava causa más frecuente de muerte para ambos sexos constituyen las enfermedades crónicas del aparato respiratorio inferior, en hombres 2,6\% ( $\mathrm{n}=$ 1038) y en mujeres $2,5 \%(n=819)$ (Instituto Nacional de Estadisticas y Censos, 2019). 
Con este panorama, se hace necesario el uso de metodologías estandarizadas para la Evaluación de los Impactos en la Salud por Contaminación Atmosférica (AP-HRA). Esta herramienta es usada por los tomadores de decisiones para la planificación de estrategias de mejoramiento del aire (WHO, 2016b). El trabajo presentado, también se ve sustentado con la evaluación de la percepción de la población con respecto a la contaminación atmosférica y sus impactos a la salud. La percepción pública de la contaminación atmosférica determina juicios y decisiones de la población que conducen a acciones cotidianas y, en consecuencia, puede determinar cierto tipo de exposición a los contaminantes (Catalán-Vasquez, 2006; Jiménez et al., 2015).

En los últimos años, una de las medidas aplicadas más recurrentes para el mejoramiento de la calidad del aire en cañones urbanos ha sido la implementación de Infraestructura Verde Urbana (IVU) debido a la promoción de políticas para la construcción de techos verdes y jardines verticales, los cuales ofrecen múltiples benefi- cios en entornos urbanos como el mejoramiento de la calidad del aire circundante, la retención de escorrentía superficial, reducciones en el efecto de isla de calor urbana y un aumento de la biodiversidad urbana (Abhijith et al., 2017; Jayasooriya et al., 2017; Pugh et al., 2012; RamírezCevallos et al., 2019; Sicard et al., 2018). Actualmente, el municipio del Distrito Metropolitano de Quito (DMQ) promueve la construcción de edificios bajo el modelo de eco-eficiencia, en los cuales la implementación de medidas de prevención del impacto ambiental en su construcción (tales como la instalación de jardines verticales), permiten el aumento de edificabilidad por sobre lo establecido en su plan de uso y ocupación del suelo original, con el fin de promover la densificación de la ciudad a lo largo de los ejes de transporte público (Herdoiza, 2017). La aplicación de un AP-HRA permite estimar los beneficios en salud de la implementación de este tipo de políticas, ya que es fundamental conocer cuánto puede disminuir la carga en salud con la aplicación de dichas acciones (Molina \& Molina, 2002). 
El objetivo de este trabajo es estudiar la percepción de la población sobre los impactos en salud asociadas a la mala calidad del aire y cuantificar mediante AP-HRA el beneficio económico y en muertes evitables (ME) debido la reducción de la concentración de contaminantes atmosféricos $\left(\mathrm{PM}_{2,5}, \mathrm{PM}_{10}\right.$ y $\left.\mathrm{O}_{3}\right)$ en ocho parroquias del DMQ debido a una agresiva aplicación de la IVU.

\section{MATERIALES Y MÉTODOS}

Para el establecimiento del escenario base, se realizaron muestreos en diez puntos de alto tráfico del DMQ durante siete días por veinticuatro horas para el ozono $\left(\mathrm{O}_{3}\right.$ en $\left.\mathrm{ppb}\right)$, y de dos días entre las 07 h00 y 19 h00 para las partículas con diámetro aerodinámico menos a 2,5 $\mu \mathrm{m}\left(\mathrm{PM}_{2,5}\right.$ en $\mu \mathrm{g}$ $\left.\mathrm{m}^{-3}\right)$ y $10 \mu \mathrm{m}\left(\mathrm{PM}_{10}\right.$ en $\left.\mu \mathrm{g} \mathrm{m}^{-3}\right)$ en cada punto, según lo descrito por Chuquer-Sola et al. (2018), y detaIlado en la Tabla 1.

Tabla 1. Concentraciones promedio de $\mathrm{PM}_{2,5}, \mathrm{PM}_{10}$ y $\mathrm{O}_{3}$ monitoreadas

\begin{tabular}{lccc}
\hline $\begin{array}{l}\text { Punto de } \\
\text { monitoreo }\end{array}$ & $\begin{array}{c}\mathrm{PM}_{2,5} \\
\left(\mu \mathrm{g} \mathrm{m}^{-3}\right)\end{array}$ & $\begin{array}{c}\mathrm{PM}_{10} \\
\left(\boldsymbol{\mu g ~ m}^{-3}\right)\end{array}$ & $\begin{array}{c}\mathrm{O}_{3} \\
(\mathrm{ppb})\end{array}$ \\
\hline PUCE & 24,0 & 72,8 & 4,9 \\
Secretaria de Seguridad & 13,4 & 41,3 & 3,7 \\
Escuela Sucre & 17,5 & 97,9 & 5,3 \\
Unidad de salud del Sur & 31,0 & 68,8 & 2,4 \\
Hogar de Paz & 27,9 & 74,2 & 2,5 \\
Teatro Capitol & 19,7 & 52,9 & 2,7 \\
Administración Calderón & 32,3 & 66,6 & 4,1 \\
Fundación Vista para todos & 13,9 & 74,1 & 3,0 \\
Colegio Gonzaga & 15,1 & 66,7 & 5,6 \\
Plaza de Cumbayá & 12,3 & 50,0 & 4,5 \\
\hline
\end{tabular}

Los muestreos de partículas fueron realizados en el interior de viviendas mientras que el $\mathrm{O}_{3}$ fue medido en el exterior, a 2,5 metros de las vías de alto tráfico. 
Las Funciones Exposición Respuesta (FER) y Riesgos Relativos (RR) para los casos estudios planteados se obtuvieron de las investigaciones multiciu- dad en el que especifica todo tipo de impactos (INECC-México, 2017) y se detallan en la Tabla 2.

Tabla 2. Funciones Exposición Respuesta y Riesgos Relativos para mortalidad general asociados con incremento de $\mathrm{PM}_{2,5}, \mathrm{PM}_{10}$ y $\mathrm{O}_{3}$ Fuente: (INECC-México, 2017)

\begin{tabular}{lcccc}
\hline Causa & Edad & RR* $^{*}$ & FER* & Ref. \\
\hline $\mathrm{PM}_{2,5}$ & $\geq 15$ & 1,006 & 0,006 & Hoek et al., 2013 \\
$\mathrm{PM}_{10}$ & $\geq 30$ & 1,0043 & 0,0043 & Künzli et al., 2000 \\
$\mathrm{PM}_{10}$ & $<1$ & 1,004 & 0,004 & Woodruff et al., 2008 \\
$\mathrm{O}_{3}$ & $\geq 30$ & 1,002 & 0,002 & Turner et al., 2016 \\
\hline
\end{tabular}

* Incremento en $1 \mu \mathrm{g} \mathrm{m}^{-3} \circ 1 \mathrm{ppb}$.

Se identificó la población expuesta a los contaminantes atmosféricos y las tasas de mortalidad para el impacto a la salud seleccionado (mortalidad general). La población bajo estudio fueron los habitantes de 8 parroquias urbanas y rurales del Distrito Metropolitano de Quito que corresponde a los 10 puntos de monitoreo. El nú- mero de habitantes fue desagregado por el grupo de edad correspondiente a las FER seleccionadas. Se determinó una línea base de salud para la población expuesta expresada en tasas basales de mortalidad general y correspondiente a las parroquias $y$ grupos etarios establecidos (Tabla 3). 


\section{Tabla 3. Mortalidad basal por parroquia y rango de edad seleccionada por cada diez mil habitantes}

Fuente (MSP, 2016)

\begin{tabular}{lccc}
\hline Parroquia & $\begin{array}{c}\text { Mortalidad } \\
<1 \text { año }\end{array}$ & $\begin{array}{c}\text { Mortalidad } \\
\geq 15 \text { años }\end{array}$ & $\begin{array}{c}\text { Mortalidad } \\
\geq 30 \text { años }\end{array}$ \\
\hline Mariscal Sucre & 486,7 & 60,6 & 87,1 \\
Centro Histórico & 48,5 & 106,6 & 171,7 \\
La Magdalena & 232,5 & 139,8 & 207,4 \\
San Juan & 521,0 & 48,3 & 73,8 \\
Itchimbia & 2746,8 & 70,6 & 106,3 \\
Calderón & 97,0 & 40,2 & 65,1 \\
Conocoto & 88,8 & 54 & 82,8 \\
Cumbayá & 73,3 & 45,1 & 67,2 \\
\hline
\end{tabular}

Existen diferentes estudios que han cuantificado la remoción de contaminantes atmosféricos que la implementación de IVU puede generar. Para el $\mathrm{PM}_{2,5}$, Jayasooriya et al. (2017) determinaron que la implementación de diversos tipos de IVU presentan un potencial de remoción anual del contaminante que oscila entre 0,96 y $1,54 \%$. Por otro lado, Nowak et. al. (2013) concluyeron que en 10 ciudades de Estados Unidos, el arbolado urbano puede generar una mejora anual de la calidad del aire en términos de remoción del contaminante de alrededor el $1 \%$. Tomando en cuenta estos antecedentes, el escenario de control seleccio- nado para el $\mathrm{PM}_{2,5}$ fue del $1 \%$ de reducción.

Con respecto al $\mathrm{PM}_{10}$ Jayasooriya et al. (2017) establecieron que la IVU podría reducir anualmente entre 32,75 y $47,95 \%$ del contaminante. Pugh et. al. (2012) determinaron que se genera un aumento en la sedimentación del material particulado en paredes verdes ubicadas en cañones de calle (zonas donde edificaciones a ambos lados de las vías no permiten un circulación del aire) de hasta el $60 \%$. Tallis et. al. (2015) siguiendo esta línea expresan que la IVU elimina entre el 1 y el $60 \%$ de las partículas reduciendo significativamente 
la exposición humana. Tomando en cuenta estos antecedentes, el escenario de control seleccionado para el $\mathrm{PM}_{10}$ fue de reducción del $30 \%$.

La reducción de los niveles $\mathrm{O}_{3}$ según Yang, McBride, Zhou \& Sun (2005) oscilan entre el $20 \%$ del total de contaminantes atmosféricos absorbidos por árboles anualmente, mientras que Jayasooriya et. al. (2017) expresan que la IVU, puede reducir entre 35,53 y $41,88 \%$ del $\mathrm{O}_{3}$. El escenario de control seleccionado para el $\mathrm{O}_{3}$ fue de reducción del $30 \%$.

Se integró la información recopilada en las anteriores etapas para el cálculo de las Muertes Evitables (ME) como resultado del cambio de concentración de $\mathrm{PM}_{2,5}, \mathrm{PM}_{10}$ y $\mathrm{O}_{3}$. Para la determinación de ME se aplicó el modelo lineal mediante la ecuación 1 de acuerdo a EPA (2008) y Echániz Pellicer et. al. (2011).

$$
\iota_{i j}=\Delta C_{j}{ }^{*} F E R_{i j} * P * T_{i}
$$

Donde $\iota_{i j}$ es el número de muertes evitables del impacto a la salud " $i$ " que se asocia al cambio de la concentración del contaminante " $j " ; \Delta C_{j}$ es el cambio de concentración del contaminante "j" que se asocia a la implementación de medidas de control; $F E R_{i j}$ corresponde a la función de exposición respuesta detallada en la Tabla 2; P corresponde a la población expuesta; $T_{i}$ corresponde tasa basal de mortalidad asociada con el impacto "i" para la población P.

Posteriormente se cuantificó el beneficio económico resultante de la estimación de las ME mediante el cálculo del Valor de una Vida Estadística (VVE) que busca monetizar el beneficio para la sociedad que implica evitar una muerte. EI VVE fue establecido siguiendo las recomendaciones de la Organización para la Cooperación y el Desarrollo Económicos (OECD, 2011) que sugiere la transferencia del VVE frecuentemente citado, producto del meta-análisis realizado por Kochi et al. (2006) de 5,4 millones de USD (a precios del año 2000), al contexto de estudio mediante el método de transferencia de beneficios que se realiza a partir de la ecuación 2 expresada por INECC-México (2017):

$$
V V E_{E C U(2018)}=V V E_{K O C H I(2000)} \times\left(\frac{I_{E C U(2000)}}{I_{U S A(2000)}}\right) \beta
$$


Donde $V V E_{E C U(2018)}$ es el Valor de una Vida Estadística (VVE) para Ecuador en dólares estadounidenses del 2018, VVE $E_{K O C H I}$ (2000) es el VVE en dólares estadounidenses del 2000 recopilado del estudio de Kochi et al. (2006), I $I_{E C U(2000)}$ es el Ingreso Nacional Bruto per cápita de Ecuador basado en el método de paridad de poder adquisitivo recopilado del Banco Mundial (2019), I USA (2000) es el Ingreso Nacional Bruto per cápita de Estados Unidos basado en el método de paridad de poder adquisitivo recopilado del Banco Mundial (2019) y $\beta$ es la elasticidad del ingreso del VVE. Se utilizaron valores de elasticidad 0,5 y 2 de acuerdo a la investigación de INECC-Mexico (2017).

También se calcularon los años de vida potencialmente perdidos (AVPP) debido a enfermedades relacionadas con la contaminación ambiental (CIE-10 códigos: I10-I15, I20-I25, C33-C34, J00-J98) con la esperanza de vida media de 75 años (Instituto Nacional de Estadisticas y Censos, 2019).

Finalmente, se realizó una encuesta de percepción de la contaminación atmosférica en las zonas de estudio. La encuesta realizada fue estructurada con diecisiete preguntas que se relacionan con: información general del encuestado, nivel de importancia del tema de contaminación atmosférica, la valorización de la calidad del aire, la relación contaminación atmosférica-salud e inferencia de enfermedades causadas por la contaminación atmosférica. Las encuestas fueron realizadas los días 29, 30 y 31 de mayo y el 8 y 19 de junio del 2019. Se aplicaron diez encuestas en los diez puntos de monitoreo especificados en la Tabla 1 generándose así un universo de cien encuestados. La población objetivo establecida fueron personas adultas que en el momento de la realización de la encuesta trabajaban o residían en las cercanías del punto de monitoreo y que, por ende, se encontraban expuestos a los niveles de contaminación registrados. Los resultados de la encuesta no se pueden considerar como generalidades para toda la población parroquial, ya que no se utilizaron criterios estadísticos para la selección del tipo de muestreo. 


\section{RESULTADOS}

La cuantificación de las Muertes Evi- fueron interpretados en función de tables se realizó mediante la aplica- los valores de las variables que sobreción de la Ecuación 1 y se muestra en salen y por ende aumentan el núla Tabla 4 y Figura 1. Los resultados mero de ME.

Tabla 4. ME basadas en el cálculo con ecuación 1 en ocho parroquias del DMQ

\begin{tabular}{lcccc}
\hline Parroquia & $\begin{array}{c}\mathrm{PM}_{2,5} \\
\geq 15 \\
\text { años }\end{array}$ & $\begin{array}{c}\mathrm{PM}_{10} \\
\mathbf{a n ̃ o s}\end{array}$ & $\begin{array}{c}\mathrm{PM}_{10} \\
\text { años }\end{array}$ & $\begin{array}{c}\geq 15 \\
\mathbf{a n ̃ o s}\end{array}$ \\
\hline Mariscal Sucre & $<1$ & 6 & 1 & $<1$ \\
Centro Histórico & $<1$ & 39 & $<1$ & 1 \\
La Magdalena & 1 & 28 & 1 & $<1$ \\
San Juan & $<1$ & 18 & 3 & $<1$ \\
Itchimbia & $<1$ & 11 & 6 & $<1$ \\
Calderón & 1 & 35 & 2 & 1 \\
Conocoto & $<1$ & 27 & 1 & 1 \\
Cumbaya & $<1$ & 7 & $<1$ & $<1$ \\
\hline
\end{tabular}




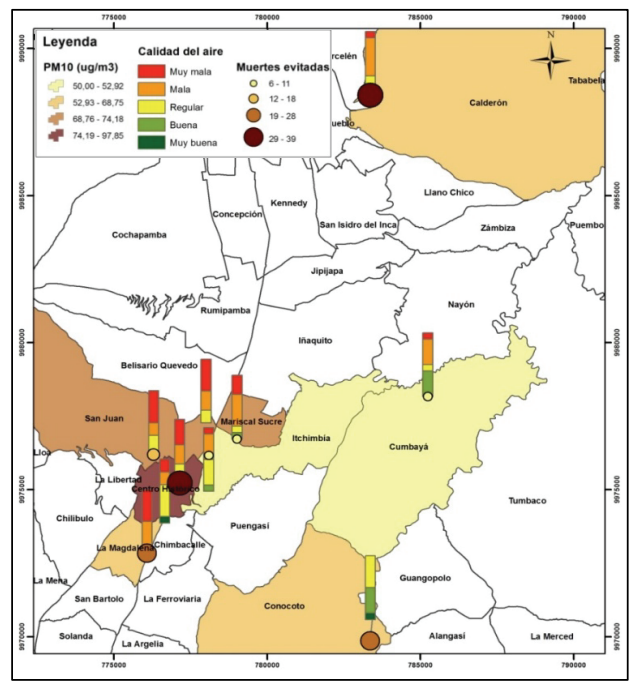

Figura 1. Contaminación, percepción y ME basadas en el cálculo con ecuación 1 en ocho parroquias del DMQ

Con respecto al cálculo del impacto económico que suponen las Muertes Evitables, se calcularon dos Valores de una Vida Estadística (VVE) a partir de la Ecuación 2, con los valores de elasticidad del ingreso de 0,5 y de 2 como escenario bajo y alto respectivamente. Se calculó la media geométrica en base a ambos resultados. Al valor resultante se lo ajustó por inflación con el incremento porcentual de los precios del año 2000 al 2018, a partir del Índice de Precios al Consumidor de USA (CPI Inflation Calculator, 2019). El resultado del VVE se muestra en la Tabla 5.
Una vez definido el VVE para Ecuador de 735111 USD a precios de 2018 se procedió a calcular la valoración económica de la ME cuyo resultado se muestra en la Tabla 6 y es de alrededor de 139,7 millones de USD. Al analizar la mortalidad existente en las parroquias estudiadas debido a enfermedades relacionadas con la contaminación del aire, se identificó que el cálculo total de Años de Vida Potencialmente Perdidos (AVPP) fue de 2515 años para ambos sexos. 
Tabla 5. Valor de una Vida Estadística para Ecuador (a precios de 2018). VVE ajustada es incluyendo la inflación desde el 2000 hasta el 2018 (45,82 \%)

\begin{tabular}{ccccc}
\hline VVE $_{\text {KOCHI (2000) }}$ & I ECU (2000) & I USA (2000) & $\begin{array}{c}\text { Media geométrica } \\
(\boldsymbol{\beta} \text { 0,5 y 2) }\end{array}$ & $\begin{array}{c}\text { VVE aj } \\
\text { (USD) }\end{array}$ \\
\hline $5{ }^{\prime} 400000$ & 6896 & 45974 & 504123 & 735112 \\
\hline
\end{tabular}

Tabla 6. Valoración económica de las Muertes evitables

\begin{tabular}{cccc}
\hline Contaminante & Grupo etario & ME & $\begin{array}{c}\text { Valoración } \\
\text { (USD) }\end{array}$ \\
\hline $\mathrm{PM}_{2,5}$ & $\geq 15$ años & 2 & $1^{\prime} 470223$ \\
$\mathrm{PM}_{10}$ & $\geq 30$ años & 171 & $125^{\prime} 704124$ \\
& $<1$ año & 14 & $10^{\prime} 291565$ \\
$\mathrm{O}_{3}$ & $\geq 30$ años & 3 & $2^{\prime} 205335$ \\
\hline & & Total & $139^{\prime} 671248$ \\
\hline
\end{tabular}

En la Figura 2 se observa un diagrama de red que resume los resultados obtenidos con respecto a la información general de los encuestados y la percepción en términos de importancia, calidad del aire e impactos a la salud. Los nodos centrales corresponden a las 10 locaciones en que fueron le- vantadas las encuestas, mientras que los nodos que los circunscriben simbolizan las respuestas. Las líneas curvas azules que unen los nodos centrales con los periféricos representan las elecciones tomadas para cada encuestado. 


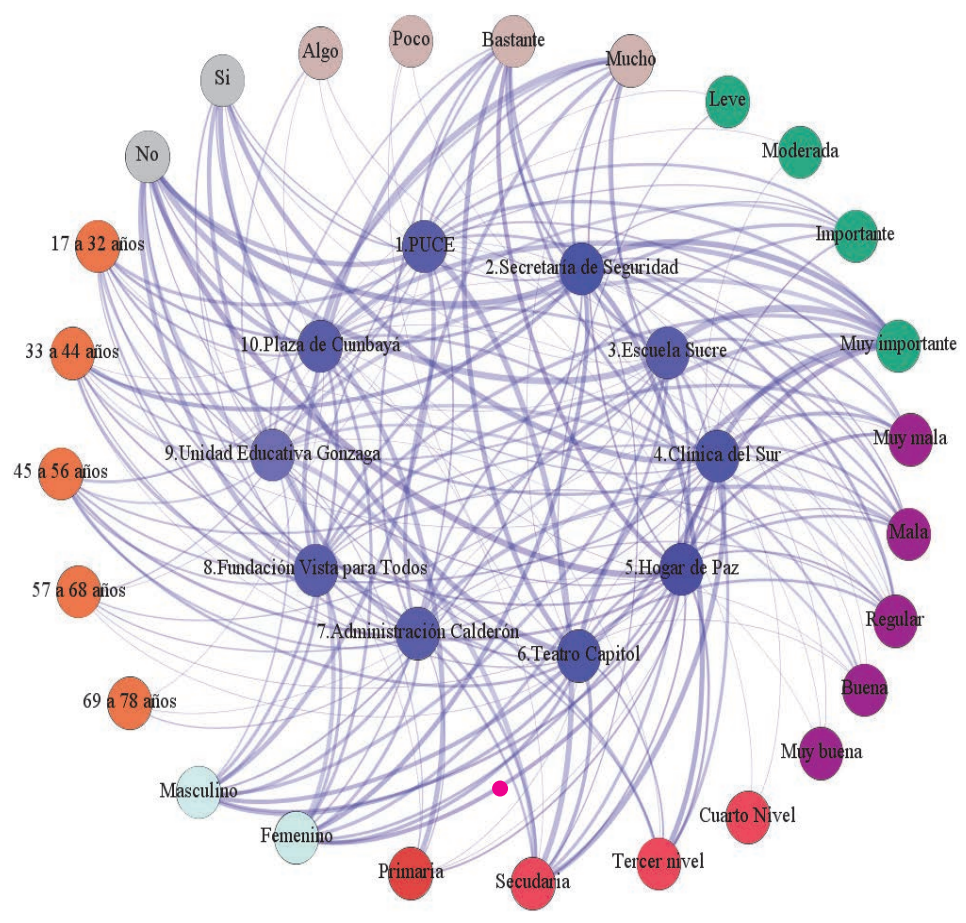

Figura 2. Diagrama de red de respuestas por lugar de encuesta.

Los colores identifican los tipos de preguntas: - Lugar de encuesta; $\bullet$ Rango de edad;

Genero; • Educación; • Percepción calidad del aire; • Nivel de importancia;

- Cuantificación de impactos en salud; Aparición de enfermedades

\section{DISCUSIÓN}

Dentro del DMQ recurrentemente se excede exceden los límites recomendados de calidad del aire para ozono $\left(\mathrm{O}_{3}\right)$, partículas menores a $2,5 \mu \mathrm{m}$ $\left(\mathrm{PM}_{2,5}\right)$, partículas menores a $10 \mu \mathrm{m}$ $\left(\mathrm{PM}_{10}\right)$ y dióxido de azufre $\left(\mathrm{SO}_{2}\right)$
(Rodas \& Arias, 2018). La ciudad cuenta con un parque automotor de cerca de quinientos mil vehículos, donde una cuarta parte de sus habitantes vive en cercanías de vías de alto tráfico (Raysoni et al., 2017) y 
está expuesta a concentraciones de contaminantes superiores a las reportadas por el sistema estatal de monitoreo (Chuquer-Sola et al., 2018). La estimación de Muertes evitables debido a un hipotético mejoramiento en la calidad del aire a filo de calle en un $30 \%$ para $\mathrm{PM}_{10}$ y $\mathrm{O}_{3}$ y $1 \%$ para $\mathrm{PM}_{2,5}$ como causa de una implementación agresiva de IVU se detalla en la Tabla 4. De estos resultados se observa que el $\mathrm{PM}_{10}$ es el contaminante cuya reducción generaría efectos más beneficiosos en la salud pública ya que se estiman $185 \mathrm{ME}$ anualmente en una población de 205530 habitantes correspondientes a las parroquias evalua- das (90 ME por cada cien mil habitantes) en los grupos etarios $<1$ año $y \geq 30$ años. El análisis espacial detallado en la Figura 1 muestra que las parroquias con mayor ME son Centro Histórico (22,8 \% del total), Calderón (20,5\% del total) y La Magdalena (16,4 \% del total), mostrando la importancia de la implementación de Infraestructura Verde Urbana de manera agresiva en estas zonas, además de la posibilidad de evitar muertes prematuras en grupos etarios relativamente jóvenes, lo que se ha demostrado por la cantidad de años de vida potencialmente perdidos (AVPP). Dentro del contexto iberoamericano existen varios estudios relacionados a esta temática descritas descritos en la Tabla 7 (Alonso Fustel et al., 2005; INECCMéxico, 2017; Ortiz-Durán \& RojasRoa, 2013).

\section{Tabla 7. Comparación de ME por cien mil habitantes}

\begin{tabular}{|c|c|c|}
\hline ME & Reducción $\mathrm{PM}_{10}$ & Fuente \\
\hline $15-20$ & $\begin{array}{c}\text { Bilbao, Madrid } \\
\text { y Sevilla, } \\
5 \mathrm{\mu g} \mathrm{m}^{-3}\end{array}$ & $\begin{array}{l}\text { (Alonso Fustel } \\
\text { et al., 2005) }\end{array}$ \\
\hline 67 & $\begin{array}{l}\text { México DF, } \\
24 \mathrm{\mu g} \mathrm{m}^{-3}\end{array}$ & $\begin{array}{l}\text { (INECC- } \\
\text { México, 2017) }\end{array}$ \\
\hline 90 & $\begin{array}{c}\text { Quito, } \\
\text { 12-29 } \mathrm{\mu g} \mathrm{m}^{-3}\end{array}$ & Este trabajo \\
\hline
\end{tabular}

Cabe recalcar que la línea base de concentraciones de partículas en este trabajo son mediciones en residencias situadas en vías de alto tráfico donde existen concentraciones de partículas superiores a las registradas por estaciones regionales de calidad del aire (Chuquer-Sola et al., 2018). Esto explica que los resultados obtenidos sean mayores a otros trabajos relacionados; sin embargo, los autores consideramos que esa evaluación proporciona una mayor aproxima- 
ción a la situación real que viven las personas dentro de sus hogares.

El cálculo de Valores de una Vida Estadística (VVE) obtenido en este trabajo se detalla en la Tabla 5 (735112 USD). En otro contexto, Ortiz-Durán \& Rojas-Roa (2013) establecieron un VVE para Bogotá de 530895 USD. Por su parte, INECC-México (2017) definió un VVE de 1,75 millones de dólares para México utilizando el método de transferencia de beneficios. Un VVE referencial a nivel internacional es el meta-análisis de Kochi et al. (2006) que define un VVE de 5,4 millones de dólares. Así, el VVE de Ecuador se muestra elevado en comparación al de Bogotá debido a diferencias metodológicas y cuestiones de cambio de moneda, sin embargo, tomando como referencia estudios de México, el VVE reportado se encuentra acorde e incluso puede llegar a ser conservador en comparación a al VVE de países con ingresos altos.

La valoración económica de los beneficios en salud que implica la reducción de la contaminación atmosférica se detalla en la Tabla 6 (139,6 millones de USD). En contraste a los resultados reportados, Martínez-Vásquez (2008) estableció para 7029 muertes evitables relacionadas a la reducción de partículas un beneficio económico de 4,8 mil millones de dólares; Ortiz-Durán \& Rojas-Roa (2013) determinaron, en localidades con hasta 21000 muertes evitables, un beneficio económico de 5,2 mil millones de dólares y el INECC-México (2017) definió un beneficio de alrededor de 7,5 mil millones de dólares por 12089 muertes evitables. La incertidumbre con que cuentan estas estimaciones determina que los resultados deben tomarse con cautela, sin embargo, se puede confirmar que los beneficios económicos de la mejora de la calidad del aire son sustanciales bajo cualquier supuesto.

Siguiendo los lineamientos teóricoprácticos propuestos por CatalánVasquez (2006) y Jiménez et al. (2015), el diagrama de red de respuestas por lugar de encuesta detallado en la Figura 2, resume los resultados obtenidos con respecto a la información general de los encuestados y su percepción en términos de relevancia del tema ambien- tal, cualificación de la calidad del aire del lugar e impactos a la salud asociados. 
Se evidencia una tendencia de percepción negativa con respecto de la calidad del aire, concentrando los resultados en las categorías de muy mala, mala y regular. En términos generales, los encuestados consideran el tema atmosférico como importante o muy importante y cuantifican que la mala calidad del aire afecta significativamente en su salud. Finalmente, no se observa una tendencia con respecto a la percepción de aparición de enfermedades asociadas.

Las recomendaciones sobre la instalación de IVU en cañones urbanos son la instalación de setos con alturas de hasta $2 \mathrm{~m}$ (radio altura / ancho $\leq$ $0,5)$ mientras que en vías amplias la calidad del aire mejora por la implementación de vegetación ancha y de baja porosidad. En ambos casos, tanto jardines verticales como jardines en techos muestran resultados satisfactorios (Abhijith et al., 2017; Ramírez-Cevallos et al., 2019). Con base en ello, el estudio presenta tres propuestas de intervención en espacios urbanos impactados con altas emisiones vehiculares en las intersecciones: a) Av. 12 de Octubre y Av. Patria; b) Av. Alonso de Angulo y Av. Vicente Maldonado; y c) calle Portoviejo y Av. América.

Dichos planteamientos obedecen a la aplicación de la matriz de sistematización RESQUECOMO, que nos permitió conocer el rol o función del espacio, las circunstancias específicas del entorno urbano, enunciar la intención o idea fuerza y definir la estrategia a implementar. Entre estas estrategias están:, la plantación del arbolado viario, implementación de jardines verticales activos y pasivos (Ramírez-Cevallos et al., 2019), y tecnologías como Hispacold eco $3^{\circledR}$, que consiste en sistemas de purificación de espacios interiores en kioskos de ventas asociados al equipamiento de transporte público, así como también el uso de sistemas de purificación de aire personal o Handtree (Su et al., 2018). La situación actual y las propuestas realizadas en este trabajo bajo el esquema RESQUECOMO se detallan en la Figura 3. 


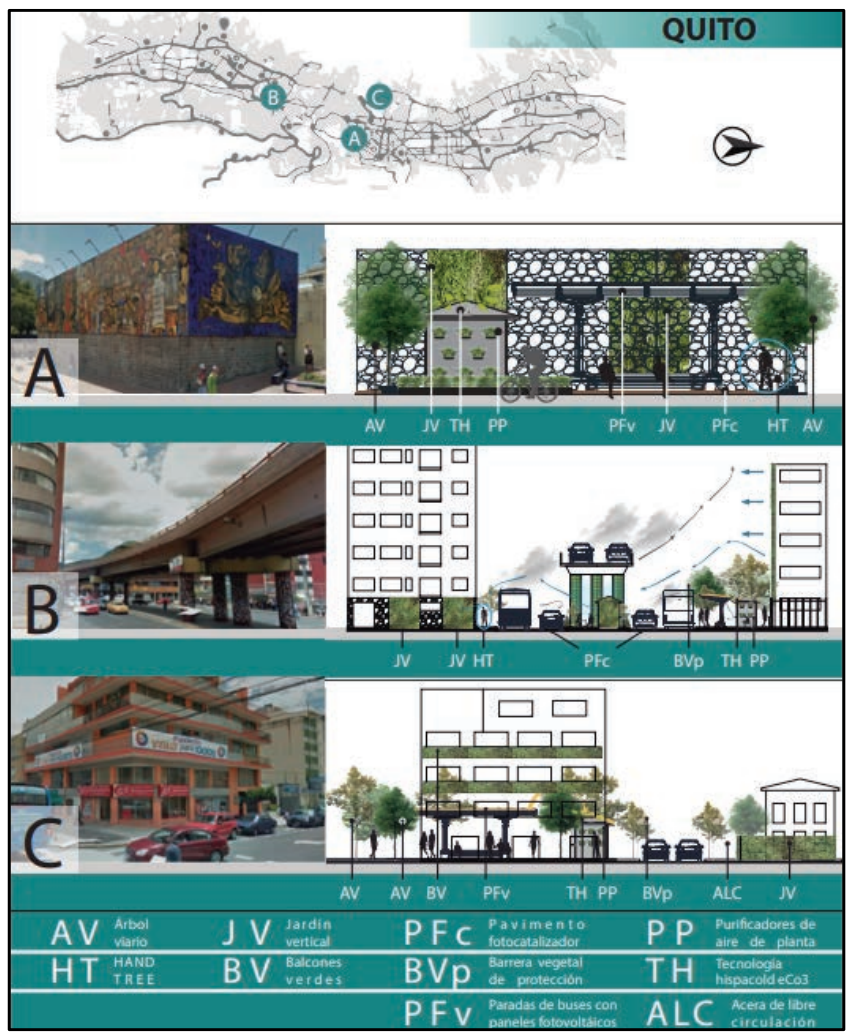

Figura 3. Situación actual y propuestas de instalación de IVU en tres zonas de alto tráfico en Quito: A) Av. 12 de Octubre y Av. Patria;

B) Av. Alonso de Angulo y Av. Vicente Maldonado; y C) calle Portoviejo y Av. América

\section{CONCLUSIÓN}

El trabajo presentado es uno de los primeros de su tipo en el Ecuador, el mismo que toma en cuenta mediciones de calidad del aire a filo de calle y en el interior de viviendas, impactadas por la contaminación de vías de alto tráfico, lo que le da un enfoque aproximado a la realidad que 
vive la ciudadanía respecto a la contaminación del aire. Mediante la metodología AP-HRA se estimaron los impactos en salud y económicos asociados a la implemen- tación agresiva de Infraestructura Verde Urbana en ocho parroquias de la ciudad de Quito, determinándose que la reducción de contaminantes $\mathrm{PM}_{10}$ (30\%), $\mathrm{O}_{3}(30 \%)$ y $\mathrm{PM}_{2,5}$ (1 \%) permitiría evitar 190 muertes anualmente en la zona de estudio, con un beneficio económico de 139,7 millones de USD. Adicionalmente, se determinó el Valor de una Vida Estadística en Ecuador (735112 USD), valor que puede servir de referencia para más estimaciones en el campo de la salud pública.

\section{AGRADECIMIENTOS}

Los autores agradecen a la Pontificia Universidad Católica del Ecuador por el financiamiento a través del proyecto O13024.

\section{LISTA DE REFERENCIAS}

Abhijith, K. V., Kumar, P., Gallagher, J., McNabola, A., Baldauf, R., Pilla, F., Broderick, B., Di Sabatino, S., \& Pulvirenti, B. (2017). Air pollution abatement performances of green infrastructure in open road and built-up street canyon environments - A review. Atmospheric Environment, 162, 71-86. https://doi.org/10.1016/j.atmosenv. 2017.05.014

Alonso Fustel, E., Martínez Rueda, T., Cambra Contín, K., Lopez Carrasco, L., Boldo Pascua, E., Zorrilla Torras, B., Daponte Codina, A., Aguilera Jiménez, I., \& Medina, S. (2005). Evaluación en cinco ciudades españoles del impacto en salud de la contaminación atmosférica por partículas. Proyecto europeo APHEIS. Revista Española de Salud Pública, 79(2), 297-308. 
Banco Mundial. (2019). INB per cápita, PPA (dólares internacionales constantes de 2011).

Catalán-Vasquez, M. (2006). Estudio de la percepción pública de la contaminación del aire y sus riesgos para la salud: perspectivas teóricas y metodológicas. Revista Del Instituto Nacional de Enfermedades Respiratorias, 19(1), 28-37. http://www.scielo. org.mx/pdf/iner/v19n1/v19n1a4.pdf

Chuquer - Sola, D., Ampudia-Vásquez, S., Puertas, C., Bustamante, L., Reina, C., \& Ramírez, F. (2018). Contaminación del aire a filo de calle en Quito, caso estudio Guayaquil y Espejo. Perfiles, 2(20), 90-99. http://ceaa.espoch.edu.ec:8080/revista.perfiles/Articuloshtml/Perfiles20Art11/Perfiles20Art11.xhtml

CPI Inflation Calculator.

Echániz Pellicer, G., Garibay Bravo, V., \& Rojas Bracho, L. (2011). Guía para evaluar los impactos en la salud por la instrumentación de medidas de control de la contaminacion atmosférica (A. M. Sanchéz Mora (ed.); 1st ed.). Instituto Nacional de Ecología - Secretaria de Medio Ambiente y Recursos Naturales.

EPA. (2008). Environmental Benefits Mapping and Analysis Program -Community Edition : User's Manual. Environmental Protection Agency.

Herdoiza, J. (2017). Instrucciones administrativas y flujos de procedimiento para aprobar el incremento de número de pisos por suelo creado por sobre lo establecido en el PUOS vigente, en el Distrito Metropolitano de Quito. http://gobiernoabierto. quito. gob.ec/Archivos/puos/Resolucion STHV-014-2017_Incremento Edificabilidad.pdf

Hoek, G., Krishnan, R. M., Beelen, R., Peters, A., Ostro, B., Brunekreef, B., \& Kaufman, J. D. (2013). Long-term air pollution exposure and cardio- respiratory mortality: a review. Environmental Health, 12(1), 43. https://doi.org/10.1186/1476-069X-1243

INECC-México. (2017). Estimación de impactos en la salud por contaminación atmosférica en la región centro del país y alternativas de gestión. http://cambioclimatico.gob.mx:8080/xmlui/handle/publicaciones/52 
Instituto Nacional de Estadisticas y Censos. (2019). Estadísticas Vitales. https://www. ecuadorencifras.gob.ec/defunciones-generales-2019/

Jayasooriya, V. M., Ng, A. W. M., Muthukumaran, S., \& Perera, B. J. C. (2017). Green infrastructure practices for improvement of urban air quality. Urban Forestry and Urban Greening, 21, 34-47. https://doi.org/10.1016/j.ufug.2016.11.007

Jiménez, M., Ferrer, A., Chaves, L., Navarro, O., Marín, J., Cárdenas, J., \& Rodríguez, S. (2015). Análisis preliminar de un cuestionario de evaluación de la percepción social de la contaminación atmosférica. REVISTA DE SALUD PÚBLICA, 17(5). https://doi.org/10.15446/rsap.v17n5.3847

Kochi, I., Hubbell, B., \& Kramer, R. (2006). An Empirical Bayes Approach to Combining and Comparing Estimates of the Value of a Statistical Life for Environmental Policy Analysis. Environmental \& Resource Economics, 34(3), 385-406. https://doi.org/ 10.1007/s10640-006-9000-8

Künzli, N., Kaiser, R., Medina, S., Studnicka, M., Chanel, O., Filliger, P., Herry, M., Horak, F., Puybonnieux-Texier, V., Quénel, P., Schneider, J., Seethaler, R., Vergnaud, J.-C., \& Sommer, H. (2000). Public-health impact of outdoor and traffic-related air pollution: a European assessment. The Lancet, 356(9232), 795-801. https://doi.org/10.1016/S0140-6736(00)02653-2

Martínez-Vásquez, S. (2008). Externalidades Ambientales Asociadas a la Contaminación del Aire [Universitat Autonoma de Barcelona]. https://dialnet.unirioja.es/servlet/ tesis?codigo=182186

Molina, L. T., \& Molina, M. J. (2002). Air Quality in the Mexico Megacity (L. T. Molina \& M. J. Molina (eds.); Vol. 2). Springer Netherlands. https://doi.org/10.1007/97894-010-0454-1

MSP. (2016). Información estadística y geográfica de salud. Mortalidad Por Grupos de Edad y Lugar de Ocurrencia 2016. https://www.salud.gob.ec/informacion-estadistica-de-produccion-de-salud/

Nowak, D. J., Hirabayashi, S., Bodine, A., \& Hoehn, R. (2013). Modeled PM2.5 removal by trees in ten U.S. cities and associated health effects. Environmental Pollution, 178, 395-402. https://doi.org/10.1016/j.envpol.2013.03.050 
OECD. (2011). Valuing Mortality Risk Reductions in Regulatory Analysis of Environmental, Health and Transport Policies: Policy Implications. https://www.oecd.org/ env/tools-evaluation/48279549.pdf

Ortiz-Durán, E. Y., \& Rojas-Roa, N. Y. (2013). Estimación de los beneficios económicos en salud asociados a la reducción de PM10 en Bogotá. Rev Salud Publica (Bogota), 15(1), 90-102. http://www.scielo.org.co/scielo.php?script=sci_arttext\&pid=S012400642013000100009

Pugh, T. A. M., MacKenzie, A. R., Whyatt, J. D., \& Hewitt, C. N. (2012). Effectiveness of Green Infrastructure for Improvement of Air Quality in Urban Street Canyons. Environmental Science \& Technology, 46(14), 7692-7699. https://doi.org/10.1021/ es300826w

Ramírez-Cevallos, F., Davis, M., Chuquer-Solá, D., \& Vallejo-Espinosa, A. (2019). CALIDAD DE AIRE EN EL CENTRO HISTÓRICO DE QUITO. Revista de Diseño Urbano \& Paisaje. DU\&P, 35(1), 50-61. http://dup.ucentral.cl/dup_35/francisco_ ramirez.pdf

Raysoni, A., Armijos, R., Weigel, M., Echanique, P., Racines, M., Pingitore, N., \& Li, W.W. (2017). Evaluation of Sources and Patterns of Elemental Composition of PM2.5 at Three Low-Income Neighborhood Schools and Residences in Quito, Ecuador. International Journal of Environmental Research and Public Health, 14(7), 674. https://doi.org/10.3390/ijerph14070674

Rodas, M., \& Arias, V. (2018). Informe de calidad del aire 2017. http://www.quitoambiente.gob.ec/ambiente/index.php/informes\#informe-calidad-del-aire-2017

Sicard, P., Agathokleous, E., Araminiene, V., Carrari, E., Hoshika, Y., De Marco, A., \& Paoletti, E. (2018). Should we see urban trees as effective solutions to reduce increasing ozone levels in cities? Environmental Pollution, 243, 163-176. https://doi.org/10.1016/j.envpol.2018.08.049

Su, Y., Liu, G., \& Zhang, L. (2018). Research on the Design of Human Settlement System and Personal Air Purification System Under the Background of Internet of Things. In Lecture Notes in Computer Science (3rd Intern, pp. 106-118). https://doi. org/10.1007/978-3-319-94370-1_8 
Tallis, M. J., Amorim, J. H., Calfapietra, C., Freer-Smith, P., Grimmond, S., \& Kotthaus, S. (2015). The impacts of green infrastructure on air quality and temperature. In D. Sinnett, N. Smith, \& S. Burgess (Eds.), Handbook on Green Infrastructure (pp. 30-49). Edward Elgar Publishing. https://doi.org/10.4337/9781783474004.00008

Turner, M. C., Jerrett, M., Pope, C. A., Krewski, D., Gapstur, S. M., Diver, W. R., Beckerman, B. S., Marshall, J. D., Su, J., Crouse, D. L., \& Burnett, R. T. (2016). Long-Term Ozone Exposure and Mortality in a Large Prospective Study. American Journal of Respiratory and Critical Care Medicine, 193(10), 1134-1142. https://doi.org/ 10.1164/rccm.201508-1633OC

WHO. (2016a). Ambient air pollution: A global assessment of exposure and burden of disease. https://www.who.int/phe/publications/air-pollution-global-assessment/en/

WHO. (2016b). Health risk assessment of air pollution - general principles. http://www. euro.who.int/_data/assets/pdf_file/0006/298482/Health-risk-assessment-air-poIlution-General-principles-en.pdf?ua $=1$

Woodruff, T. J., Darrow, L. A., \& Parker, J. D. (2008). Air Pollution and Postneonatal Infant Mortality in the United States, 1999-2002. Environmental Health Perspectives, 116(1), 110-115. https://doi.org/10.1289/ehp.10370

Yang, J., McBride, J., Zhou, J., \& Sun, Z. (2005). The urban forest in Beijing and its role in air pollution reduction. Urban Forestry \& Urban Greening, 3(2), 65-78. https://doi.org/10.1016/j.ufug.2004.09.001 